\title{
A Tale of Ellipsoids in Potential Theory
}

\section{Dmitry Khavinson and Erik Lundberg}

\section{Dirichlet's Problem}

Let us start our story with the Dirichlet problem. This problem of finding a harmonic function in a, say, smoothly bounded domain $\Omega \subset \mathbb{R}^{n}$ matching a given continuous function $f$ on $\partial \Omega$ gained huge attention in the second half of the nineteenth century due to its central role in Riemann's proof of the existence of a conformal map of any simply connected domain onto the disk. Later on, Riemann's proof was criticized by Weierstrass, and, after considerable turmoil, it was corrected and completed by Hilbert and Fredholm; cf. [27] for a very nice historical account. Here we want to focus on algebraic properties of solutions to the Dirichlet problem when $\Omega$ is an ellipsoid and the data $f$ possess nice algebraic properties. Thus, we first present the following proposition.

Proposition 1. Consider the ellipsoid

$$
\Omega=\left\{x \in \mathbb{R}^{n}: \sum_{j=1}^{n} \frac{x_{j}^{2}}{a_{j}^{2}}-1 \leq 0\right\},
$$

where $a_{1} \geq a_{2} \geq \cdots \geq a_{n}>0$. The solution $u$ to the Dirichlet problem

$$
\left\{\begin{array}{l}
\Delta u=0 \quad \text { in } \Omega \\
\left.u\right|_{\partial \Omega}=p
\end{array}\right.
$$

Dmitry Khavinson is professor of mathematics at the University of South Florida. His email address is dkhavins@cas. usf.edu.

Erik Lundberg is Golomb Assistant Professor of mathematics at Purdue University. His email address is e1 undber@math. purdue.edu.

The first author acknowledges support from the NSF grant DMS-0855597.

DOI: http://dx.doi.org/10.1090/noti1082 where $p$ is a polynomial of $n$ variables, is a harmonic polynomial. Moreover,

$$
\operatorname{deg} u \leq \operatorname{deg} p .
$$

Remark 1. Proposition 1 was widely known in the nineteenth century for $n=2,3$ (perhaps due to Lamé) and was proved with the use of ellipsoidal harmonics. It is still widely known nowadays for balls but often disbelieved for ellipsoids. The first author has won a substantial number of bottles of cheap wine betting on its truthfulness at various math events and then producing the following proof that was related to him by Harold S. Shapiro. The idea of the proof goes back at least to Fischer [11]; we do not know who thought of it first, but we hope the reader will agree that this proof deserves to be called, following P. Erdôs, the "proof from the book."

Proof. Denote by $P_{n, m}=P_{m}$ the finite-dimensional space of polynomials of degree less than or equal to $m$ in $n$ variables. Let $q(x)=\sum \frac{x_{j}^{2}}{a_{j}^{2}}-1$ be the defining quadratic for $\partial \Omega$. Consider the linear operator $T$ : $P_{m} \rightarrow P_{m}$ defined by

$$
T(r):=\Delta(q r) .
$$

The maximum principle yields at once that $\operatorname{ker} T=$ 0 , so $T$ is injective. Since $\operatorname{dim} P_{m}<\infty$, this implies that $T$ is surjective.

Hence, given $P \in P_{m}$ with $m \geq 2$, we can find a polynomial $r \in P_{m-2}$ such that $\operatorname{Tr}=\Delta P$. The function

$$
u=P-q r
$$

is then the solution of (1). 
Proposition 1 was extended [20] to the case of entire data. Namely, entire data $f$ (i.e., an entire function of variables $x_{1}, x_{2}, \ldots, x_{n}$ ) yields an entire solution to the Dirichlet problem in ellipsoids. This result was sharpened by Armitage in [1], who showed that the solution's order and type are dominated by that of the data.

One might get bold at this point and ask if Proposition 1 extends to, say, rational or algebraic data; i.e., does a smooth data function in (1) that is a rational (algebraic) function of $x_{1}, x_{2}, \ldots, x_{n}$ imply rational (algebraic) solution $u$ ? The answer is a resounding "no", but the proofs become technically more involved; see [3].

\section{The Dirichlet Problem, Ellipsoids, and Bergman Orthogonal Polynomials}

It was conjectured in [20] that Proposition 1 (without the degree condition (2)) characterizes ellipsoids. Recently, using "real Fischer spaces", H. Render confirmed this conjecture for many algebraic surfaces [28]. In two dimensions, the conjecture was confirmed under a degree-related condition on the solution in terms of the data [21]. This utilized a surprising equivalence, established by M. Putinar and N. Stylianopoulos [26], of the conjecture to the existence of finite-term recurrence relations for Bergman orthogonal polynomials. In order to state the degree conditions and the associated recurrence conditions, assume that $\Omega$ is a domain in $\mathbb{R}^{2}$ with $C^{2}$-smooth boundary. Let $\left\{p_{m}(z)\right\}$ be the Bergman orthogonal polynomials (orthogonal with respect to area measure over $\Omega$ ). These are analytic polynomials of the complex variable $z$. Consider the following properties for $\Omega$.

(a) There exists $C$ such that for a polynomial data of degree $m$ there always exists a polynomial solution of the Dirichlet problem posed on $\Omega$ of degree less than or equal to $m+C$.

(b) There exists $N$ such that for all $k, m$, the solution of the Dirichlet problem with data $\bar{Z}^{k} Z^{m}$ is a harmonic polynomial of degree $\leq(N-1) k+m$ in $z$ and of degree less than or equal to $(N-1) m+k$ in $\bar{z}$.

(c) There exists $N$ such that $\left\{p_{m}\right\}$ satisfy a (finite) $(N+1)$-recurrence relation; i.e., there are constants $a_{m-j, m}$ such that

$$
\begin{aligned}
z p_{m}= & a_{m+1, m} p_{m+1}+a_{m, m} p_{m} \\
& +\cdots+a_{m-N+1, m} p_{m-N+1} .
\end{aligned}
$$

(d) The Bergman orthogonal polynomials of $\Omega$ satisfy a finite-term recurrence relation; i.e., for every fixed $\ell>0$, there exists an $N(\ell)>0$, such that $\left\langle z p_{m}, p_{\ell}\right\rangle=0$, $m \geq N(\ell)$. (e) For any polynomial data there exists a polynomial solution of the Dirichlet problem posed on $\Omega$.

Properties (d) and (e) are essentially equivalent [26], and (a) $\Rightarrow(\mathrm{b}),(\mathrm{b}) \Leftrightarrow(\mathrm{c})$, and (c) $\Rightarrow(\mathrm{d})$. In [21] the authors used ratio asymptotics of orthogonal polynomials to show that (b) and equivalently (c) each characterize ellipses. The weaker statement that (a) characterizes ellipsoids was proved in arbitrary dimensions [22]. For more about the Khavinson-Shapiro conjecture stated in [20], we refer the reader to [21], [17], [22], [26], [28], and the references therein.

\section{The Mean Value Property for Harmonic Functions}

The mean value property for harmonic functions can be rephrased as saying that the average of any harmonic function over concentric balls is a constant. As we formulate precisely below, there is a mean value property for ellipsoids which says the average of any harmonic function over confocal ellipsoids is a constant.

Consider a heterogeneous ellipsoid

$$
\Gamma:=\left\{x \in \mathbb{R}^{N}: \sum_{j=1}^{N} \frac{x_{j}^{2}}{a_{j}^{2}}-1=0\right\},
$$

where $a_{1}>a_{2}>\cdots>a_{N}>0$, and let $\Omega$ be its interior.

Definition. A family of ellipsoids $\left\{\Gamma_{\lambda}\right\}$,

$$
\Gamma_{\lambda}=\left\{x \in \mathbb{R}^{N}: \sum_{j=1}^{N} \frac{x_{j}^{2}}{a_{j}^{2}+\lambda}-1=0\right\},
$$

where $-a_{N}^{2}<\lambda<+\infty$, is called a confocal family (for $N=2$ these are ellipses with the same foci).

Note that the shapes of confocal ellipsoids differ; as $\lambda \rightarrow \infty, \Gamma_{\lambda}$ looks like a sphere, and when $\lambda \rightarrow-a_{N}^{2}$,

$\Gamma_{\lambda} \rightarrow\left\{x \in \mathbb{R}^{N}: x_{N}=0, \sum_{j=1}^{N-1} \frac{x_{j}^{2}}{a_{j}^{2}-a_{N}^{2}}-1 \leq 0\right\}=: E$.

$E$ is called the focal ellipsoid.

The following classical theorem goes back to Maclaurin, who considered prolate spheroids in $\mathbb{R}^{3}\left(a_{1}>a_{2}=a_{3}\right)$. General ellipsoids were treated later by Laplace [23, Chapter 2].

Theorem 1. Let $u$ be an entire harmonic function. Then

$$
\frac{1}{\left|\Omega_{\lambda}\right|} \int_{\Omega_{\lambda}} u(x) d x=\text { const. }
$$

for all $\lambda: \lambda>-a_{N}^{2}$.

From now on, for the sake of brevity, we shall only consider the case $N \geq 3$. 


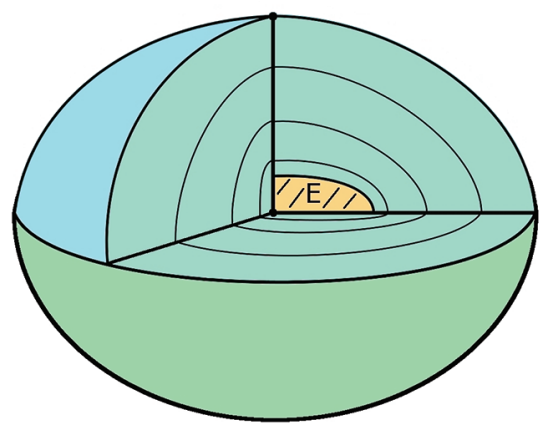

Figure 1. The mean value over confocal ellipsoids is constant.

Remark 2. Maclaurin's theorem is a corollary (via a simple change of variables; see [5, Chapter VI, Section 16] or [17, Chapter 13]) of the following result of Ásgeirsson: Suppose $u=u(x, y)$, where $x \in \mathbb{R}^{m_{1}}$, $y \in \mathbb{R}^{m_{2}}$ satisfy the ultrahyperbolic equation

$$
\Delta_{x} u=\Delta_{y} u \text {. }
$$

Then, if $\mu_{i}(x, y, r), i=1,2$, denote, respectively, the mean values of $u$ over $m_{i}$-dimensional balls of radius $r$ centered at $(x, y)$, we have $\mu_{1}(x, y, r)=$ $\mu_{2}(x, y, r)$.

Here we offer a purely algebraic approach to Maclaurin's theorem [17, Chapter 13]. The following notions are due to E. Fischer [11] (see also [31, Chapter IV]). Let $H_{k}$ be the space of homogeneous polynomials of degree $k$. If $f \in H_{k}$, then (using the standard multi-index notation of L. Schwartz [1], [31])

$$
f(z)=\sum_{|\alpha|=k} f_{\alpha} z^{\alpha} .
$$

Introduce an inner product on $H_{k}$ (called the Fischer inner product), by letting

$$
\left\langle z^{\alpha}, z^{\beta}\right\rangle= \begin{cases}0, & \alpha \neq \beta, \\ \alpha !, & \alpha=\beta .\end{cases}
$$

If $f=\sum_{|\alpha|=k} f_{\alpha} Z^{\alpha}, g=\sum_{|\alpha|=k} g_{\alpha} Z^{\alpha}$, then $\langle f, g\rangle=$ $\sum_{|\alpha|=k} a ! f_{\alpha} \overline{g_{\alpha}}$. With respect to the Fischer inner product, the operators $\left(\frac{\partial}{\partial z}\right)^{\alpha}$ and multiplication by $z^{\alpha}$ are adjoint. Also, it follows from the definition (4) that $\frac{1}{m !}(z \cdot \bar{\xi})^{m}$ is a reproducing kernel for $H_{m}$; i.e., for all $f \in H_{m}$,

$$
\frac{1}{m !}\left\langle f,(z \cdot \bar{\xi})^{m}\right\rangle=f(\xi) .
$$

Indeed, it is enough to check this for monomials, and for all multi-indices $\alpha,|\alpha|=m$, we see that

$$
\begin{gathered}
\frac{1}{m !}\left\langle z^{\alpha},(z \cdot \bar{\xi})^{m}\right\rangle=\frac{1}{m !}\left\langle z^{\alpha}, \sum_{|\beta|=m} \frac{m !}{\beta !} z^{\beta} \bar{\xi}^{\beta}\right\rangle \\
=\frac{1}{\alpha !}\left\langle z^{\alpha}, z^{\alpha} \bar{\xi}^{\alpha}\right\rangle=\xi^{\alpha} .
\end{gathered}
$$

Let $\mathcal{H}_{m} \subset H_{m}$ denote the space of harmonic polynomials of degree $m$.

Lemma 1. The linear span of harmonic polynomials $(z \cdot \bar{\xi})^{m}$ for all $\xi \in \Gamma_{0}=\left\{\xi \in \mathbb{C}^{N}: \sum_{j=1}^{N} \xi_{j}^{2}=0\right\}$ (the isotropic cone) equals $\mathcal{H}_{m}$.

Proof of Lemma 1. Let us assume for the sake of contradiction that there is a nonzero polynomial $u \in \mathcal{H}_{m}$ satisfying

$$
\left\langle u,(z \cdot \bar{\xi})^{m}\right\rangle=0, \quad \forall \xi \in \Gamma_{0} .
$$

Using the reproducing kernel condition (5), we have $u(\xi)=0$ for all $\xi \in \Gamma_{0}$. By Hilbert's Nullstellensatz

$$
u(\xi)=\left(\sum_{j=1}^{N} \xi_{j}^{2}\right) q(\xi), \text { for some } q \in H_{m-2} .
$$

But then, since $u$ is harmonic, we have

$$
0=\langle\Delta u, q\rangle=\left\langle u,\left(\sum_{j=1}^{N} \xi_{j}^{2}\right) q\right\rangle=\langle u, u\rangle,
$$

where we have used the fact that multiplication and differentiation are adjoint. Hence, $u \equiv 0$.

Proof of Maclaurin's theorem. It suffices to check (3) for harmonic homogeneous polynomials, and in view of Lemma 1, we just have to check it for polynomials

$$
(z \cdot \bar{\xi})^{m}, \quad \xi \in \Gamma_{0} .
$$

Fix $\lambda$. Let $b_{i}=\left(a_{i}^{2}+\lambda\right)^{1 / 2}$ be the semiaxes of $\Omega_{\lambda}$. We have to show that

$$
\begin{array}{r}
\frac{1}{|\Omega|} \int_{\Omega}(x \cdot \bar{\xi})^{m} d x=\frac{1}{\left|\Omega_{\lambda}\right|} \int_{\Omega_{\lambda}}(x \cdot \bar{\xi})^{m} d x, \\
\forall \xi \in \Gamma_{0} .
\end{array}
$$

Changing variables in both integrals $x_{k}=a_{k} y_{k}$, $x_{k}=b_{k} y_{k}$, we see that it suffices to show the following:

(6)

$$
\int_{B}\left(\sum_{k=1}^{N} a_{k} y_{k} \overline{\xi_{k}}\right)^{m} d y=\int_{B}\left(\sum_{k=1}^{N} b_{k} y_{k} \overline{\xi_{k}}\right)^{m} d y,
$$

where $B$ is the unit ball in $\mathbb{R}^{N}$. Since $\xi \in \Gamma_{0}$ implies that

$$
\sum_{k=1}^{N}\left(\left(a_{k} \bar{\xi}_{k}\right)^{2}-\left(b_{k} \bar{\xi}_{k}\right)^{2}\right)=\overline{-\lambda^{2} \sum_{k=1}^{N} \xi_{k}^{2}}=0,
$$

verifying (6) reduces to checking the following assertion.

Assertion. The polynomial

$$
P(t):=\int_{B}\left(\sum_{k=1}^{N} x_{k} t_{k}\right)^{m} d x
$$


depends only on $\sum_{k=1}^{N} t_{k}^{2}$, for $t \in \mathbb{C}^{N}$.

The assertion follows from the rotation invariance of $P$ [17, Chapter 13].

The following application is noteworthy. Let $\Omega$ be an ellipsoid with semiaxes $a_{1}>a_{2}>\cdots>a_{N}>0$, and let

$$
u_{\Omega}(x):=C_{N} \int_{\Omega} \frac{d y}{|x-y|^{N-2}}, \quad x \in \mathbb{R}^{N} \backslash \Omega
$$

be the exterior potential of $\Omega$.

As above, $E$ denotes the focal ellipsoid. The following corollary of Maclaurin's theorem describes a so-called mother body [14], i.e., a measure supported inside the ellipsoid which generates the same gravitational potential (outside the ellipsoid) as the uniform density but is minimally supported in some sense (see the discussion in [14]). In this case the mother body is supported on $E$, a set of codimension one with connected complement.

Corollary 1. For $x \in \mathbb{R}^{N} \backslash \bar{\Omega}$

$$
u_{\Omega}(x)=C_{N} \int_{E} \frac{d \mu(y)}{|x-y|^{N-2}},
$$

where

$$
\begin{aligned}
d \mu(y)= & 2\left(\prod_{j=1}^{N} a_{j}\right)\left(\prod_{j=1}^{N-1}\left(a_{j}^{2}-a_{N}^{2}\right)\right)^{-1 / 2} \\
& \times\left.\left(1-\sum_{j=1}^{N-1} \frac{y_{j}^{2}}{a_{j}^{2}-a_{N}^{2}}\right)^{1 / 2} d y^{\prime}\right|_{E}
\end{aligned}
$$

( $d y^{\prime}$ is Lebesgue measure on $\left\{y_{N}=0\right\}$ ).

Sketch of proof. Since the integrand is harmonic, we have by MacLaurin's theorem

$$
u_{\Omega}(x)=\frac{\prod_{j=1}^{N} a_{j}}{\prod_{j=1}^{N}\left(a_{j}^{2}+\lambda\right)^{1 / 2}} \int_{\Omega_{\lambda}} \frac{C_{N}}{|x-y|^{N-2}} d y .
$$

After simplifying this integral using Fubini's theorem, the corollary is established by applying the Lebesgue dominated convergence theorem as $\lambda \rightarrow$ $-a_{N}^{2}[17$, Chapter 13].

We note in passing that finding relevant mother bodies for oblate and prolate spheroids (supported on a disk and segment, respectively) could be a satisfying exercise.

Since the density of the distribution $d \mu$ is real analytic in the interior of $E$ (viewed as a set in $\mathbb{R}^{N-1}$ ), we note the following corollary:

Corollary 2. The potential $u_{\Omega}(x)$ extends as a (multivalued) harmonic function into $\mathbb{R}^{N} \backslash \partial E$.

An extension of this fact and a "high ground" view of the mother body, based on holomorphic PDE in $\mathbb{C}^{n}$, is discussed in the section "The Cauchy Problem: A View from $\mathbb{C}^{n}$.

\section{The Equilibrium Potential of an Ellipsoid. Ivory's Theorem}

Considering that force is the gradient of potential, the following theorem, due to Newton, can be paraphrased in a rather catchy way: "there is no gravity in the cavity".

Theorem 2 (Newton's theorem). Let $t>1$, and consider the ellipsoidal shell $S:=t \Omega \backslash \Omega$ between two homothetic ellipsoids. The potential $U_{S}$ of uniform density on $S$ is constant inside the cavity $\Omega$.

In fact, ellipsoids are characterized by this property; i.e., Newton's theorem has a converse [7], [8], [24], [17]. A modern approach to Newton's theorem and far-reaching generalizations due to V. I. Arnold and A. Givental are sketched in the epilogue.

A consequence of Newton's theorem is that the gravitational potential $U_{\Omega}$ of $\Omega$ is a quadratic polynomial inside $\Omega$. Namely,

$$
U_{\Omega}(x)=B-\sum_{i=1}^{N} A_{j} x_{j}^{2}, \quad \text { for } x \in \Omega,
$$

with $B=C_{N} \int_{\Omega} \frac{d V(y)}{|y|^{N-2}}=U_{\Omega}(0)$, where $C_{N}=\frac{1}{\operatorname{Vol}\left(S^{N-1}\right)}$. Indeed, denoting by $\Omega_{t}=t \Omega$ (for $t>1$ ) the dilated ellipsoid, one computes that its gravitational potential is $u_{t}(x)=t^{2} u(x / t)$. Since Newton's theorem implies that (where $u$ is the potential of the original ellipsoid) $u_{t}-u=$ const inside $\Omega$, the smaller ellipsoid, then taking partial derivatives $\partial^{\alpha}$, with respect to $x,|\alpha|=2$, yields that $\partial^{\alpha} u_{t}(x)=$ $\partial^{\alpha} u(x / t)=\partial^{\alpha} u(x)$. Thus all these partial derivatives are homogeneous of degree zero inside $\Omega$. They are also obviously continuous and, hence, are constants, thus yielding $U_{\Omega}$ to be a quadratic as claimed.

Denoting $\Gamma:=\partial \Omega$, consider the single layer potential

$$
V(x)=C_{N} \int_{\Gamma} \frac{\rho(y)}{|x-y|^{N-2}} d A(y),
$$

where $\rho(y)$ is a mass density and $d A(y)$ on $\Gamma$ is the surface area measure. Also, $V(x)$ is called an equilibrium potential if $V(x) \equiv 1$ on $\Gamma$ and hence inside $\Omega$. We again focus on the case $N \geq 3$. The quantity

$$
\sigma:=\lim _{|x| \rightarrow \infty}|x|^{N-2} V(x)=C_{N} \int_{\Gamma} \rho(y) d A(y)
$$

is called capacity.

On the way to proving Ivory's theorem, we note an explicit formula for the equilibrium potential.

Corollary 3. With $B$ as above, in $\mathbb{R}^{N} \backslash \bar{\Omega}$, we have

$$
V(x)=\frac{1}{B}\left(\hat{\mu}-\frac{1}{2} \sum_{i=1}^{N} x_{i} \frac{\partial \hat{\mu}}{\partial x_{i}}\right),
$$


where $\hat{\mu}(x)=C_{N} \int_{E} \frac{d \mu\left(y^{\prime}\right)}{\left|x-y^{\prime}\right|^{N-2}}, y^{\prime}=\left(y_{1}, y_{2}, \ldots, y_{N-1}\right.$, $0)$, and $d \mu\left(y^{\prime}\right)$ is the MacLaurin quadrature measure supported on the focal ellipsoid E (cf. Corollary 1).

Proof. Thus the right-hand side of (7) is harmonic in $\mathbb{R}^{N} \backslash \bar{\Omega}$ (in fact, in $\mathbb{R}^{N} \backslash E$ ) since $\hat{\mu}$ is harmonic there and $\Delta(x \cdot \nabla \hat{\mu})=n \Delta \hat{\mu}=0$. On $\Gamma$, by Maclaurin's theorem and Newton's theorem,

$$
\hat{\mu}=U_{\Omega}(x)=B-\sum_{i=1}^{N} A_{j} x_{j}^{2} .
$$

Moreover, since $U_{\Omega}(x)$ has continuous first derivatives throughout $\mathbb{R}^{N}$, we can differentiate (8) on $\Gamma$ and thus obtain

$$
\begin{aligned}
& \frac{1}{B}\left(\hat{\mu}-\frac{1}{2} \sum_{i=1}^{N} x_{i} \frac{\partial \hat{\mu}}{\partial x_{i}}\right) \\
& \quad=\frac{1}{B}\left(B-\sum_{i=1}^{N} A_{j} x_{j}^{2}+\frac{1}{2} \sum_{i=1}^{N} 2 A_{j} x_{j}^{2}\right)=1 .
\end{aligned}
$$

Thus, the right-hand side of (7) equals $V(x)$ on $\Gamma$. Both functions are harmonic in $\mathbb{R}^{N} \backslash \bar{\Omega}$ and vanish at infinity, and the statement follows.

Corollary 4 (Ivory's theorem). The equipotential surfaces of the equilibrium potential $V(x)$ are confocal with $\Gamma$.

For the proof, one simply notes that the righthand side of (7) changes only by a constant factor when $\Omega$ is replaced by a confocal ellipsoid

$$
\Omega_{\lambda}:=\left\{x: \sum^{N} \frac{x_{j}^{2}}{a_{j}^{2}+\lambda} \leq 1, \lambda \geq 0\right\} .
$$

Namely, $B \rightarrow B_{\lambda}$ while $\frac{d \mu_{\lambda}}{d \mu}=\frac{\operatorname{Vol}\left(\Omega_{\lambda}\right)}{\operatorname{Vol}(\Omega)}$.

For the classical proof of Ivory's theorem, see [23], [12, Lecture 30].

\section{Ellipsoids in Fluid Dynamics}

Let us pause for a moment to mention applications of these properties of ellipsoids to two problems in fluid dynamics. In the first problem, involving a slowly moving interface, viscosity plays an important role. In the second problem, viscosity is completely neglected, while vorticity plays the dominant role.

\section{Moving Interfaces and Richardson's Theorem}

Imagine a blob of incompressible viscous fluid within a porous medium surrounded by an inviscid fluid. Suppose there is a sink at position $x_{0}$ in the region $\Omega_{t}$ occupied by viscous fluid, so $\Omega_{t}$ is shrinking with time. Darcy's law governs the fluid velocity $v$ in terms of the pressure $P$ :

$$
v=-\nabla P \text {. }
$$

Incompressibility implies that

$$
\nabla \cdot v=-\Delta P=0
$$

except at the sink $x_{0}$. The pressure of the inviscid fluid is assumed constant. Neglecting surface tension (a rather controversial assumption), the pressure matches at the interface, which gives a constant (say, zero) boundary condition for $P$, so $P$ is nothing more than the harmonic Green's function with a singularity at $x_{0}$. The mathematical problem is then to track the evolution of a domain $\Omega_{t}$ whose boundary velocity is determined by the gradient of its own Green's function. See [32] for an engaging exposition of the two-dimensional case of this problem.

Given a harmonic function $u(x)$, Richardson's theorem [29] describes the time dependence of the integration of $u$ over the domain occupied by the viscous fluid. In the language of integrable systems this represents "infinitely many conservation laws".

Theorem 3 (S. Richardson, 1972). Let $u(x)$ be a function harmonic in $\Omega_{t}$ for all $t$. Then

$$
\frac{d}{d t} \int_{\Omega_{t}} u(x) d V(x)=-Q u\left(x_{0}\right),
$$

where $x_{0}$ is the position of the sink with pumping rate $Q>0$.

An alternative setup places the viscous fluid in an unbounded domain with a single sink at infinity [7]; a reformulation of Richardson's theorem implies that the potential inside the cavity of the shell regions $\Omega_{t} \backslash \Omega_{s>t}$ is constant. Thus it is a consequence of Newton's theorem (Theorem 2) that an increasing family of homothetic ellipsoids is an exact solution. In fact, this is the only solution starting from a bounded inviscid fluid domain that exists for all time and fills the entire space [7].

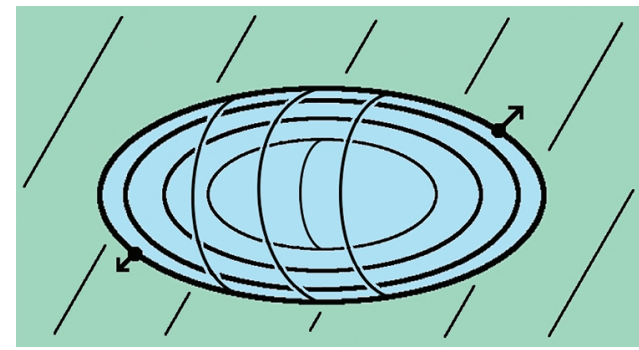

Figure 2. Viscous fluid occupies the exterior. The ellipsoid grows homothetically.

Returning to the case when the viscous fluid is bounded, suppose the initial domain $\Omega_{0}$ is an ellipsoid and consider the problem of determining sinks and pumping rates such that $\left\{\Omega_{t}\right\}_{t=0}^{T}$ shrinks to zero volume as $t \rightarrow T$. As a consequence of the mean value property, one can solve this problem exactly, thus removing all of the fluid, that is, provided we can stretch our imaginations to 
allow a continuum of sinks (spread over the focal set $E$ ). Starting from the given ellipsoid $\Omega_{0}$, the evolution $\Omega_{t}$ is a family of ellipsoids confocal to $\Omega_{0}$ shrinking down to the (zero-volume) focal set $E$. The pumping rate is given by the time-derivative of the quadrature measure appearing in Corollary 1.

\section{The Quasigeostrophic Ellipsoidal Vortex Model}

Based on the observation that motion in the atmosphere is roughly stratified into horizontal layers, the quasigeostrophic approximation provides a simplified version of the Euler equations (governing inviscid incompressible flow). Further assumptions reduce the entire dynamics to a scalar field, the potential vorticity, which in the high Reynolds number limit forms coherent regions of uniform density. Even with these simplifications, the problem can still be quite complicated. For instance, approximating the regions of potential vorticity by clouds of point-vortices, one encounters the notoriously difficult $n$-body problem.

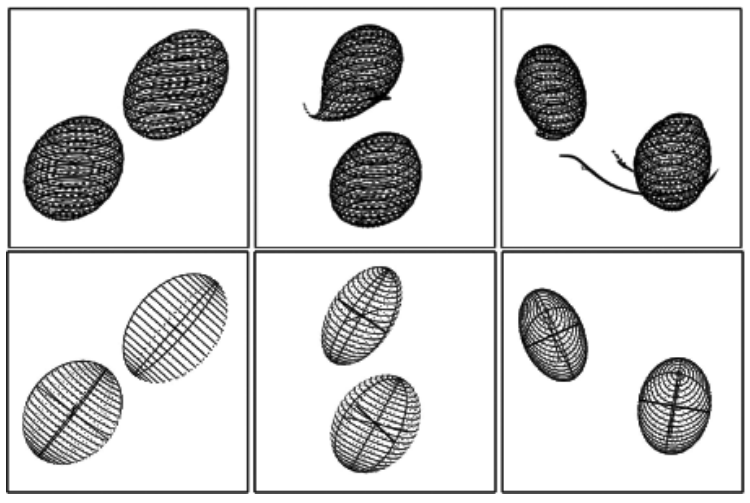

Figure 3. Top row: A vortex simulation using "contour dynamics". Bottom row: A faster, but still accurate, simulation using the ellipsoidal vortex model.

The quasigeostrophic ellipsoidal vortex model developed by Dritschel, Reinaud, and McKiver [9] simulates the interaction of ellipsoidal regions of vorticity (see Figure 3, included here with the kind permission of Dritschel, Reinaud, and McKiver). As these regions interact, the length and alignment of semiaxes can change, but nonellipsoidal deformations are filtered out. (Note that a single ellipsoidal vortex is stable for a certain range of axis ratios.) The effect that one ellipsoid has on another is determined by its exterior potential, and thus the mean value property can be used to replace the ellipsoid by a two-dimensional set of potential vorticity on its focal ellipse (with density determined by Corollary 1) which can be further approximated by point vortices.

Remark 3. It is interesting to single out the twodimensional case of the moving interface problem

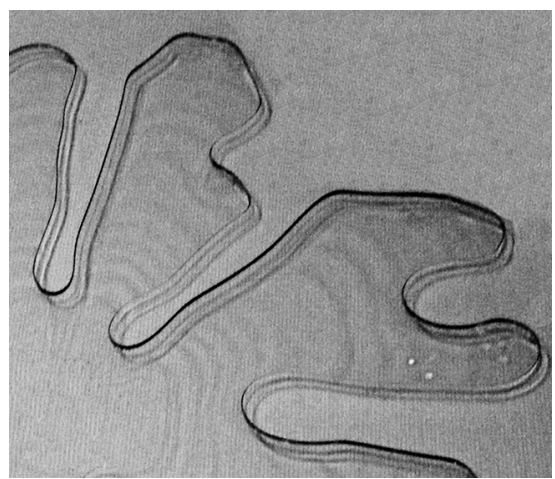

Figure 4. Viscous fingering in a Hele-Shaw cell.

which serves as a model for viscous fingering in a Hele-Shaw cell. ${ }^{1}$ Conformal mapping techniques lead to explicit exact solutions that can even exhibit the tip-splitting depicted in Figure 4. The vortex dynamics problem also admits many sophisticated analytic solutions in the two-dimensional case [6]. For a compelling survey discussing quadrature domains as a common thread linking these and several other fluid dynamics problems, see [6].

\section{The Cauchy Problem: A View from $\mathbb{C}^{n}$}

The problem mentioned in the section "The Mean Value Property for Harmonic Functions" of analytically continuing the exterior potential $U_{\Omega}$ inside the region $\Omega$ occupied by mass was studied by Herglotz [15] and can be reformulated as studying the singularities of the solution to the following Cauchy problem posed on the initial surface $\Gamma:=\partial \Omega$ :

$$
\left\{\begin{array}{l}
\Delta M=1, \quad \text { near } \Gamma, \\
M \equiv_{\Gamma} 0,
\end{array}\right.
$$

where the notation $M \equiv_{\Gamma} G$ indicates that $M$ along with its gradient coincide with $G$ and its gradient, respectively, on $\Gamma$.

The fact that $M$ carries the same singularities in $\Omega$ as the analytic continuation $u$ of $U_{\Omega}$ is a consequence of the fact that $u$ itself is given by the piecewise function

$$
u:=\left\{\begin{array}{l}
U_{\Omega}, \text { outside } \Omega, \\
U_{\Omega}-M, \text { inside } \Omega .
\end{array}\right.
$$

The reason is that $u$ is harmonic on both sides of $\Gamma$ and is $C^{1}$-smooth across $\Gamma$. (Note that, inside $\Omega, U_{\Omega}$ denotes the physical gravitational potential which solves a Poisson equation $\Delta U_{\Omega}=1$.) An extension

\footnotetext{
${ }^{1}$ A Hele-Shaw cell is a lab apparatus consisting of two closely spaced sheets of glass with a small hole in the top piece; after filling the gap with viscous fluid, one may inject a bubble of less viscous fluid. This experiment is cheap and easy to perform-in fact the photograph in Figure 4 was taken in the second author's home.
} 
of Morera's theorem (attributed to S. Kovalevskaya) implies that $u$ is actually harmonic across $\Gamma$, i.e., $\Gamma$ is a removable singularity set for $u$. Thus $u$ is the desired analytic continuation of $U_{\Omega}$ across $\Gamma$, and the singularities of $u$ in $\Omega$ are carried by $M$.

Further reformulating the problem, note that the so-called Schwarz potential of $\Gamma, W=\frac{1}{2}|x|^{2}-M$, has the same singularities as $M$ and solves a Cauchy problem for Laplace's equation:

$$
\left\{\begin{array}{l}
\Delta W=0 \quad \text { near } \Gamma \\
W \equiv_{\Gamma} \frac{1}{2}|\chi|^{2} .
\end{array}\right.
$$

This is a rather delicate (ill-posed according to Hadamard) problem, and our discussion of it will pass from $\mathbb{R}^{n}$ to the complex domain $\mathbb{C}^{n}$. Let us first consider a more intuitive Cauchy problem for a hyperbolic equation where similar behavior can be observed while staying in the real domain. Explicitly, consider

$$
\left\{\begin{array}{l}
v_{x y}=1 \text { near } \gamma \\
v \equiv_{\gamma} 0
\end{array}\right.
$$

where $\gamma$ is, say, a real analytic curve in $\mathbb{R}^{2}$.

For hyperbolic equations the mantra is "singularities propagate along characteristics." If the solution is singular at some point $\left(x_{0}, y_{0}\right)$, then one can trace the source of this singularity back to $\gamma$ by following the characteristic cone with vertex at $\left(x_{0}, y_{0}\right)$. One expects to find a singularity in the data itself at a point where this cone intersects $\gamma$, but what if the data function has no singularities as in (14)? It is still possible for a singularity to propagate to the point $\left(x_{0}, y_{0}\right)$ if the characteristic cone from $\left(x_{0}, y_{0}\right)$ is tangent to $\gamma$. The point of tangency is called a characteristic point of $\gamma$.

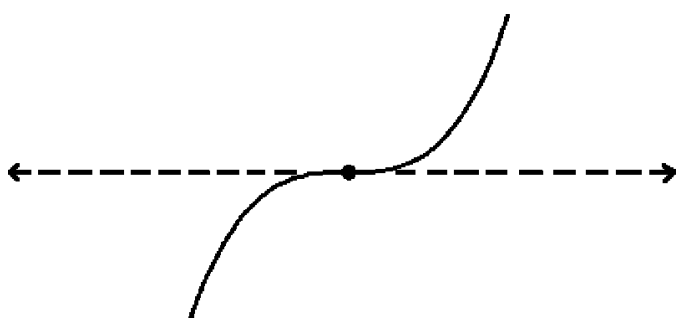

Figure 5. The solution to (14) is regular except on the tangent characteristic $\{y=0\}$.

For example, suppose $\gamma:=\left\{y=x^{3}\right\}$. We can solve (14) exactly:

$$
v(x, y)=x \cdot y-\frac{x^{4}}{4}-\frac{3}{4} y^{4 / 3} .
$$

The solution is singular on the characteristic $\{y=0\}$ which is tangent to the initial curve $\gamma$ at the point $(0,0)$; see Figure 5 .

The singularities in the solution of (13) also propagate along tangent characteristics. The important difference is that the characteristic points (the "birth places" of singularities) reside on the complexification of $\Gamma$, the complex hypersurface given by the same defining equation.

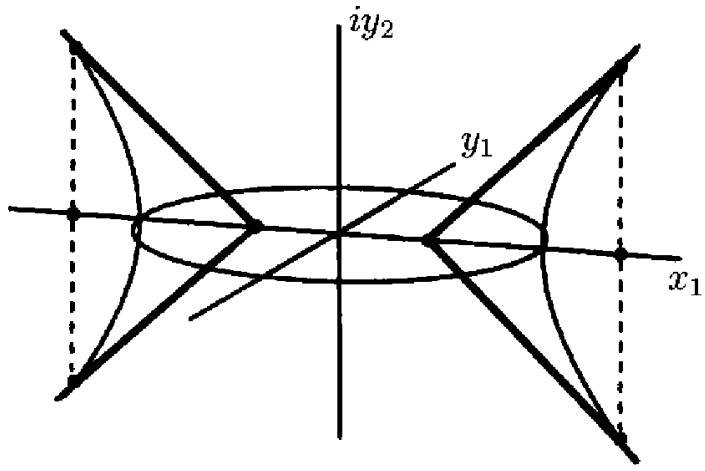

Figure 6. The characteristic lines tangent to $\Gamma$ at four characteristic points intersect $\mathbb{R}^{2}$ precisely at the foci.

For ellipsoids, these ideas can be made precise. Namely, the following result, due to G. Johnsson [16], was proved using a globalization of Leray's principle, a local theory governing propagation of singularities.

Theorem 4 (G. Johnsson, [16]). All solutions of the Cauchy problem (13) with entire data $f$ on $\Gamma:=\left\{z \in \mathbb{C}^{n}: \sum_{1}^{n} z_{1}^{2} / a_{i}^{2}=1\right\}$ extend holomorphically along all paths in $\mathbb{C}^{n}$ that avoid the characteristic surface $\Sigma$ (consisting of all characteristic lines tangent to $\Gamma$ ).

The intersection $\Sigma \cap \mathbb{R}^{n}=E$ is the focal ellipsoid that was discussed in previous sections. This provides, according to the properties of the Schwartz potential discussed above, a $\mathbb{C}^{n}$-explanation of a rather physical fact that $E$ supports a measure solving an inverse potential problem. As Johnsson notes, there is an unexpected coincidence between potential-theoretic foci (points where singularities of $W$ are located) and algebraic foci in the classical sense of Plücker [16]. Understanding this correspondence and extending it to higher-degree algebraic surfaces is part of a program advocated by the first author and H. S. Shapiro. The case $n=2$ is more transparent, but for $n>2$ it is virtually unexplored.

\section{Epilogue}

Newton's theorem can be reformulated in terms of a single layer potential obtained by shrinking a constant-density ellipsoidal shell to zero thickness (while rescaling the constant), leading to a nonconstant density $\rho(x)=1 /|\nabla q(x)|$, where $q(x)$ is the defining quadratic of the ellipsoid. This is sometimes called the standard single layer potential (it is different from the equilibrium potential discussed in the section "The Equilibrium Potential of an 
Ellipsoid. Ivory's Theorem"). The modern approach due to V. I. Arnold and, then, A. Givental [2], [13], views the force at $x_{0}$ induced by infinitesimal charges at two points $x_{1}, x_{2}$ on a line $\ell$ through $x_{0}$ as a sum of residues for a contour integral in the complex extension $L$ of $\ell$. The vanishing of force then follows from deforming the contour to infinity. The detailed proof can be found in [17, Chapter 14].

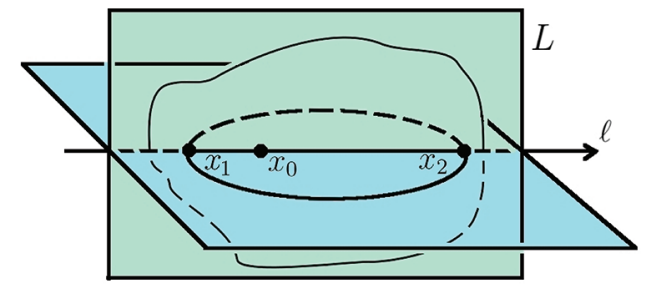

Figure 7. The force from two points is realized as a sum of residues in the complex line $L$.

The same proof can be used to extend Newton's theorem beyond ellipsoids to any domain of hyperbolicity of a smooth, irreducible real algebraic variety $\Gamma$ of degree $k$. A domain $\Omega$ is called a domain of hyperbolicity for $\Gamma$ if for any $x_{0} \in \Omega$, each line $\ell$ passing through $x_{0}$ intersects $\Gamma$ at precisely $k$ points. For example, the interior of an ellipsoid is a domain of hyperbolicity, and if a hypersurface of degree $2 k$ consists of an increasing family of $k$ ovaloids, then the smallest one is the domain of hyperbolicity.

Defining the standard single layer density on $\Gamma$ in exactly the same way as before, except that the sign + or - is assigned on each connected component of $\Gamma$ depending on whether the number of obstructions for "viewing" this component from the domain of hyperbolicity of $\Gamma$ is even or odd, the Arnold-Givental generalization of Newton's theorem implies, in particular, that the force due to the standard layer density vanishes inside the domain of hyperbolicity (cf. [2], [13] for more general statements and proofs).

As a final remark, returning to ellipsoids, and even taking $n=2$, let us note an application to gravitational lensing of Corollary 1 . The twodimensional version of Maclaurin's theorem plays a key role in formulating analytic descriptions for the gravitational lensing effect for certain elliptically symmetric lensing galaxies [10], [4] (cf. [19], [25] for terminology). Here the projected mass density that is constant on confocal ellipses produces at most four lensed images [10]. The density that is constant on homothetic ellipses produces at most six images [4]. In connection to the converse to Newton's theorem, whenever the rare focusing effect in gravitational lensing produces a continuous "halo" (a.k.a. Einstein ring; cf. [19] for some striking NASA pictures) around the lensing galaxy (of any shape), the "halo" necessarily turns out to be either a circle or an ellipse [10]. But this alley leads to the beginning of another story. Note: Due to considerations of space, the reference list has been shortened. The more complete list of references is available in [18].

\section{References}

1. D. H. ARMitAge, The Dirichlet problem when the boundary function is entire, J. Math. Anal. Appl. 291 (2004), no. 2, 565-577.

2. V. I. ARNOLD, Magnetic analogs of the theorems of Newton and Ivory, Uspekhi Mat. Nauk 38 (1984), no. 5, 253-254 (Russian).

3. S. R. Bell, P. EBENFElt, D. KHAVInSON, and H. S. SHAPIRO, On the classical Dirichlet problem in the plane with rational data, J. d'Analyse Math. 100 (2006), 157-190.

4. W. BERGWEILER and A. EREMENKo, On the number of solutions of a transcendental equation arising in the theory of gravitational lensing, Comput. Methods Funct. Theory 10 (2010), 303-324.

5. R. CourAnT and D. HiLbert, Methods of Mathematical Physics. Vol. II: Partial Differential Equations, Interscience Publishers (a division of John Wiley \& Sons), New YorkLondon, 1962.

6. D. CROWDY, Quadrature domains and fluid dynamics, Oper. Thy.: Adv. and Appl. 156 (2005), 113-129.

7. E. DiBEnEDETTO and A. FriedMAN, Bubble growth in porous media, Indiana Univ. Math. J. 35 (1986), 573-606.

8. P. Dive, Attraction des ellipsoides homogènes et réciproques d'un théorème de Newton, Bull. Soc. Math. France 59 (1931), 128-140.

9. D. G. DRITSChel, J. N. ReINAUd, and W. J. MCKIVER, The quasi-geostrophic ellipsoidal vortex model, J. Fluid Mech. 505 (2004), 201-223.

10. C. D. FAssnacht, C. R. Keeton, and D. KhavinSON, Gravitational lensing by elliptical galaxies and the Schwarz function, in Trends in Complex and Harmonic Analysis, B. Gustafsson and A. Vasilev (eds.), Birkhäuser, 2007, 115-129.

11. E. FISCHER, Über die Differentiationsprozesse der Algebra, J. für Math. 148 (1917), 1-78.

12. D. FuCHS and S. TABACHNIKOV, Mathematical Omnibus: Thirty Lectures on Classic Mathematics, Amer. Math. Soc., Providence, RI, 2007.

13. A. B. GIVENTAL, Polynomiality of electrostatic potentials, Uspekhi Mat. Nauk 39 (1984), no. 5, 253-254 (Russian).

14. B. GUSTAFSSON, On mother bodies of convex polyhedra, SIAM J. Math. Anal. 29 (1998), 1106-1117.

15. G. Herglotz, Über die analytische Fortsetzung der Potentials ins Innere der anziehenden Massen, Preisschr. der Jablonowski Gesellschaft 4 (1914).

16. G. JoHnsson, The Cauchy problem in $\mathbb{C}^{n}$ for linear second order partial differential equations with data on a quadric surface, Trans. Amer. Math. Soc. 344, no. 1 (1994), 1-48.

17. D. KHAVInson, Holomorphic Partial Differential Equations and Classical Potential Theory, Universidad de La Laguna Press, 1996.

18. D. KHAVINSON and E. LUNDBERG, A tale of ellipsoids in potential theory, with extended list of references, 2013, http://arxiv.org/abs/1309.2042. 


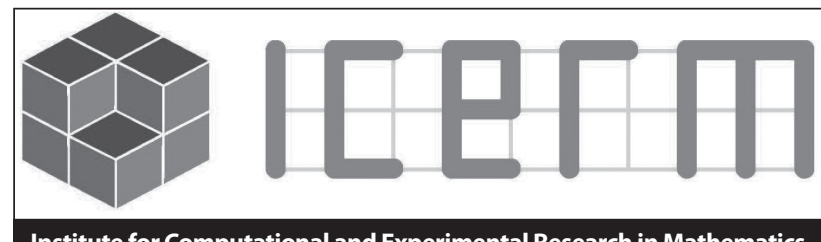

Institute for Computational and Experimental Research in Mathematics

\section{SEMESTER PROGRAM: SPRING 2015}

\section{Phase Transitions and Emergent Properties}

February 2 - May 8, 2015

\section{Organizing Committee:}

Mark Bowick, Syracuse University

Beatrice de Tiliere, Université Pierre et Marie Curie, Paris

Richard Kenyon, Brown University

Charles Radin, University of Texas at Austin

Peter Winkler, Dartmouth College

\section{Program Description:}

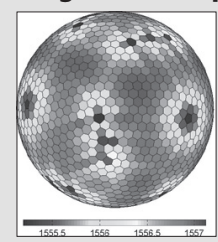

Emergent phenomena are properties of a system of many components which are only evident or even meaningful for the collection as a whole. A typical example is a system of many molecules, whose bulk properties may change from those of a fluid to those of a solid in response to changes in temperature or pressure. The basic mathematical tool for understanding emergent phenomena is the variational principle, most often employed via entropy maximization. The difficulty of analyzing emergent phenomena, however, makes empirical work essential; computations generate conjectures and their results are often our best judge of the truth. The semester will concentrate on different aspects of current interest, including unusual settings such as complex networks and quasicrystals, the onset of emergence as small systems grow, and the emergence of structure and shape as limits in probabilistic models.

Workshops:

- Crystals, Quasicrystals and Random Networks February 9-13, 2015

- Small Clusters, Polymer Vesicles and Unusual Minima March 16-20, 2015

- Limit Shapes April 13-17, 2015
19. D. Khavinson and G. NeumanN, From the fundamental theorem of algebra to astrophysics: A "harmonious" path, Notices Amer. Math. Soc. 55, no. 6 (2008), 666-675.

20. D. KHAVINSON and H. S. SHAPIRO, Dirichlet's problem when the data is an entire function, Bull. London Math. Soc. 24 (1992), 456-468.

21. D. KHAVINSON and N. STYLIANOPOUlos, Recurrence relations for orthogonal polynomials and the KhavinsonShapiro conjecture, in Around the Research of Vladimir Maz'ya II, Partial Differential Equations, A. Laptev, (ed.), International Mathematical Series, Vol. 12, Springer, 2010, pp. 219-228,

22. E. LUNDBERG and H. RENDER, The Khavinson-Shapiro conjecture and polynomial decompositions, J. Math. Analysis Appl. 376 (2011), 506-513.

23. W. D. MacMillan, The Theory of the Potential, Dover Publications, Inc., New York, 1958.

24. W. NiKLIBORC, Eine Bemerkung über die Volumpotentiale, Math. Zeitschrift 35 (1932), 625-631.

25. A. O. PetTERS, Gravity's action on light, Notices Amer. Math. Soc. 57 (2010), 1392-1409.

26. M. PUTINAR and N. STYLIANOPOULOS, Finite-term relations for planar orthogonal polynomials, Complex Anal. Oper. Theory 1 (2007), no. 3, 447-456.

27. C. ReID, Hilbert, Springer-Verlag, New York, 1996.

28. H. RENDER, Real Bargmann spaces, Fischer decompositions and sets of uniqueness for polyharmonic functions, Duke Math. J. 142 (2008), 313-352.

29. S. RichARDSON, Hele-Shaw flows with a free boundary produced by the injection of fluid into a narrow channel, J. Fluid Mech. 56 (1972), 609-618.

30. H. S. ShapIRO, The Schwarz Function and Its Generalizations to Higher Dimensions, Wiley-Interscience, 1992.

31. E. M. STEIN and G. WEISS, Introduction to Fourier Analysis on Euclidean Spaces, Princeton Univ. Press, Princeton NJ, 1971.

32. A. N. VARCHENKO and P. I. ETINGOF, Why the Boundary of a Round Drop Becomes a Curve of Order Four, University Lecture Series, Vol. 3, Amer. Math. Soc., Providence, RI, 1992.

\section{Program details:} http://icerm.brown.edu
ICERM welcomes applications for long- and short-term visitors. Support for local expenses may be provided. Full consideration will be given to applications received by March 17, 2014. Decisions about online workshop applications are typically made 1-3 months before each program, as space and funding permit. ICERM encourages women and members of underrepresented minorities to apply.
About ICERM: The Institute for Computational and Experimental Research in Mathematics is a National Science Foundation Mathematics Institute at Brown University in Providence, Rhode Island. Its mission is to broaden the relationship between mathematics and computation.

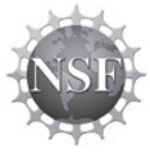

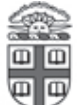
BROWN 